\title{
Can a single floating body be expressed as the sum of two bodies?
}

\author{
Hiroshi KAGEMOTO, The University of Tokyo, J apan \\ Motohiko MURAI, Yokohama National University, J apan \\ Masashi KASHIWAGI, Kyushu University, J apan
}

\section{Introduction}

As a numerical tool for the hydroelastic analysis of a very large floating structure (VLFS), Murai \& Kagemoto (1999) have proposed a method in which the structure is divided into small substructures and the substructures are treated as if they are independent freely floating structures while the structural rigidity that constraints their motions are accounted for as additional restoring forces in the equations of motion of each substructure. In this process, the hydrodynamic forces acting on the substructures are evaluated while accounting for the hydrodynamic interactions among them by the hydrodynamic interaction theory of Kagemoto \& Yue (1986).

When the VLFS is a pontoon-type structure, however, there arise some ambiguities in applying the hydrodynamic interaction theory for the evaluation of the hydrodynamic forces acting on the substructures.

\section{The ambiguities in applying the hydrodynamic interaction theory}

For the sake of simplicity, we consider the hydrodynamic interactions of two sub-bodies that comprise a single body as shown in Figure 1.

In the hydrodynamic interaction theory of Kagemoto \& Yue (1986), the ambient scattering wave field around each body in incident waves of angular frequency $\omega$ is expressed as the summation of cylindrical waves in the local cylindrical coordinate system $\left(r_{i}, \theta_{i}, z_{i}\right)(i=1,2)$ fixed to the corresponding sub-body as follows.

$$
\phi_{i}\left(r_{i}, \theta_{i}, z_{i}\right)=\frac{\cosh k\left(z_{i}+h\right)}{\cosh k h} \sum_{n=-\infty}^{\infty} A_{0 n} H_{n}{ }^{(1)}\left(k r_{i}\right) e^{i n \theta_{i}}+\sum_{m=1}^{\infty} \cos k_{m}\left(z_{i}+h\right) \sum_{n=-\infty}^{\infty} A_{m n} K_{n}\left(k_{m} r_{i}\right) e^{i n \theta_{i}}
$$

Here the velocity potential that represents the wave field is assumed to be written as $\operatorname{Re}\left(\phi_{i} e^{-i \omega t}\right) . H_{n}{ }^{(1)}, K_{n}$ are respectively the $n$ th-order Hankel functions of the first kind and modified Bessel functions of the second kind. $k, k_{m}(m=1,2, \cdots)$ are the positive real roots of the following dispersion equations for water depth $h$ and gravitational acceleration $g$.

$$
k \tanh k h=\omega^{2} / g, \quad k_{m} \tan k_{m} h=-\omega^{2} / g
$$


In the hydrodynamic interaction theory, the scattering waves due to Sub-body 1 given by Equation (1) ( $i=1)$ is treated as additional incident waves to Sub-body 2 and vice versa. The ambiguities then arise are summarized as the following 2 points.

\section{Question-1}

Is it justified to treat as if there exists some water between the sub-bodies although there actually exists no water between them?

There is no water between the two sub-bodies.

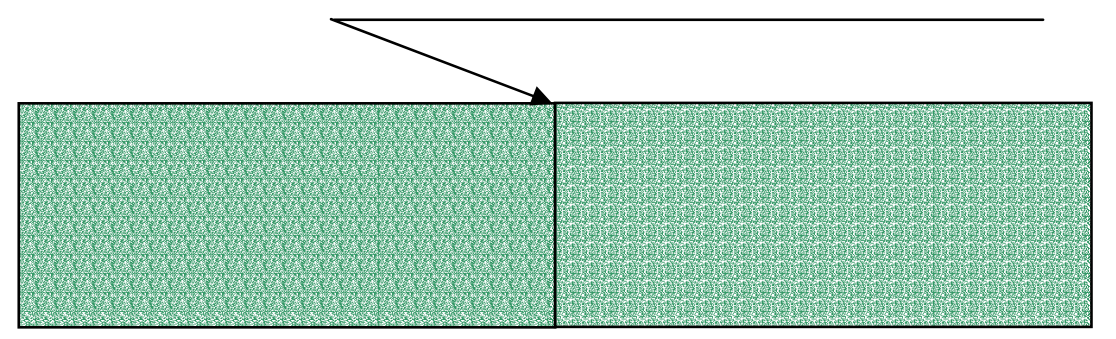

Sub-body 1

Sub-body 2

Figure $1 \mathrm{~A}$ single body as the sum of two sub-bodies

\section{Question-2}

Can the wave field due to the wave scattering by Sub-body 1 be expressed by Equation (1) everywhere in the vicinity of Sub-body 2 ?

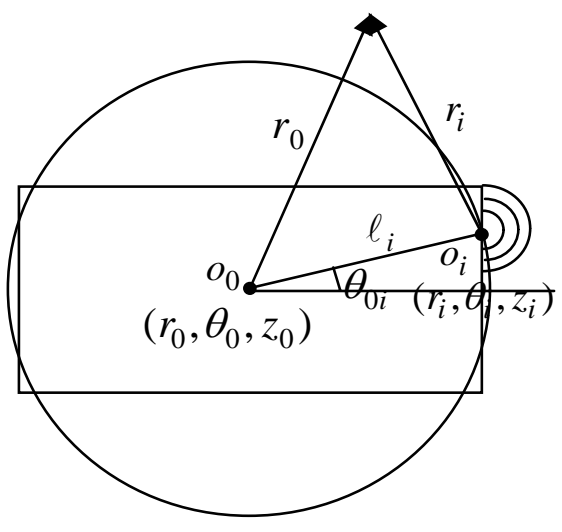

Figure 2 Representation of the cylindrical waves emanating from the distributed singularities by the cylindrical waves emanating from the origin of the coordinate system common to all the singularities

Perhaps, Question-2 may need some explanation.

It is well known that the wave field around any (floating/submerged) body can be expressed by the hydrodynamic singularities distributed over the wetted surface of the corresponding body. In other words, the ambient wave field $\phi$ around any body can be expressed by the summation of the cylindrical wave field emanating from each 
hydrodynamic singularity as follows.

$$
\phi=\sum_{i=1}^{M}\left[\frac{\cosh k\left(z_{i}+h\right)}{\cosh k h} \sum_{n=-\infty}^{\infty} A_{0 n_{i}} H_{n}{ }^{(1)}\left(k r_{i}\right) e^{i n \theta_{i}}+\sum_{m=1}^{\infty} \cos k_{m}\left(z_{i}+h\right) \sum_{n=-\infty}^{\infty} A_{m n_{i}} K_{n}\left(k_{m} r_{i}\right) e^{i n \theta_{i}}\right]
$$

Here $M$ represents the number of hydrodynamic singularities distributed over the body surface and $\left(r_{i}, \theta_{i}, z_{i}\right)$ represents the local cylindrical coordinate system fixed to the i-th singularity. The question is, whether the wave field represented by Equation (2) can al ways be expressed in terms of the cylindrical waves emanating from a certain common single coordinate system fixed to the corresponding body as in Equation (1). According to the addition theorem of Bessel functions, the following relationship holds.

$$
\sum_{n=-\infty}^{\infty} A_{0 n_{i}} H_{n}{ }^{(1)}\left(k r_{i}\right) e^{i n \theta_{i}}=\sum_{n=-\infty}^{\infty} B_{m n_{i}} H_{n}{ }^{(1)}\left(k r_{0}\right) e^{i n \theta_{0}}
$$

Here $B_{m n_{i}}$ is given as

$$
B_{m n_{i}} \equiv \sum_{m=-\infty}^{\infty}(-1)^{n+m} A_{0 m_{i}} J_{m-n}\left(k \ell_{i}\right) e^{i(m-n) \theta_{0 i}}
$$

where $\ell_{i}, \theta_{0 i}$ are defined as shown in Figure 2. Substituting Equation (3) into Equation (2), the first term of $\phi$ can now be written as follows.

$$
\sum_{i=1}^{M}\left[\frac{\cosh k\left(z_{i}+h\right)}{\cosh k h} \sum_{n=-\infty}^{\infty} A_{0 n_{i}} H_{n}{ }^{(1)}\left(k r_{i}\right) e^{i n \theta_{i}}\right]=\frac{\cosh k\left(z_{0}+h\right)}{\cosh k h} \sum_{n=-\infty}^{\infty}\left(\sum_{i=1}^{M} B_{m n_{i}}\right) H_{n}{ }^{(1)}\left(k r_{0}\right) e^{i n \theta_{0}}
$$

Considering $\sum_{i=1}^{M} B_{m n_{i}}$ as $A_{0 n}$, it seems now that Equation (2) can really be re-written in terms of the single common coordinate system $\left(r_{0}, \theta_{0}, z_{0}\right)$. In other words, the wave field resulted as the sum of cylindrical waves emanating from $M$ singularities may be expressed by the cylindrical waves emanating from the origin of the single common coordinate system.

Here, on the other hand, care must be taken on the fact that the addition theorem of Bessel functions given by Equation (3) holds only if $r_{0}>\ell_{i}$. This implies that the wave field due to i-th singularity can not be expressed properly in terms of the $\left(r_{0}, \theta_{0}, z_{0}\right)$ coordinate system in the vicinity of the body where $r_{0}<\ell_{i}$. Therefore, the wave field inside the circumcircle of the largest horizontal cross-section of the body may not be 
expressed properly as the sum of cylindrical waves emanating from the origin of the cylindrical coordinate system fixed to the center of the circumcircle.

\section{Conclusions}

Numerical and theoretical investigation on the two questions raised in the previous section were carried out. Although the details of the investigation will be presented in the workshop, here the conclusions obtained are described.

\section{Answer to Question-1}

It seems to be justified to treat as if there exists some water between the sub-bodies although there actually exists no water between them.

Answer to Question-2

In the strict sense of mathematics, it can not be justified to evaluate the hydrodynamic forces acting on a single body by dividing the single body into two sub-bodies and applying the hydrodynamic interaction theory, if the largest horizontal circumcircle of one of the sub-bodies contains part of the other sub-body as shown in Figure 3.

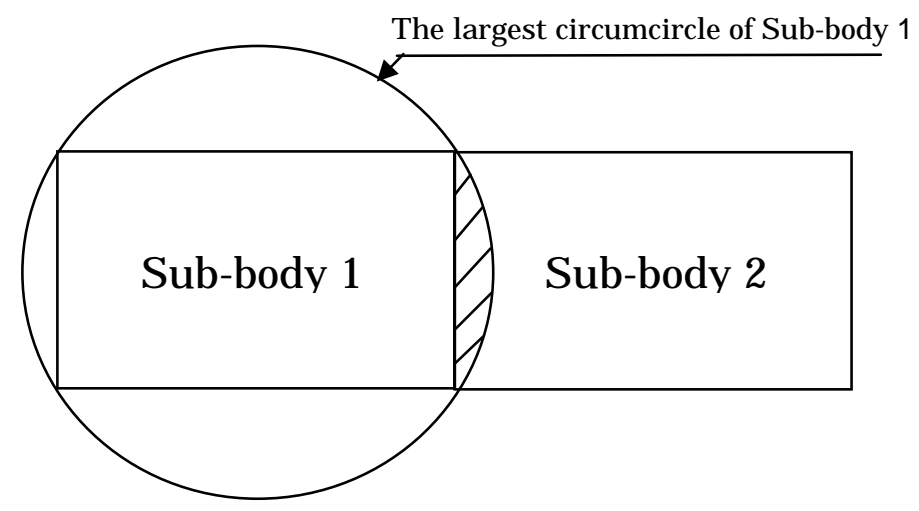

Figure 3 An example in which the largest circumcircle of one sub-body contains part of the other sub-body

In practice, however, the errors caused by violating the prerequisite of the Bessel function's addition theorem may be very small for the evaluation of horizontal forces whereas they could be of a noticeable amount for the evaluation of vertical forces.

\section{References}

1. M. Murai, H. Kagemoto and M. Fujino, J. Marine Science and Technology, Vol.4, No.3, 1999.

2. H. Kagemoto and D. K.P. Yue, J. Fluid Mech., Vol.166, 1986. 


\section{Discusser: D.V. Evans}

The method you describe was used some time ago and called the wide-spacing approximation in a paper by M. Srokosz \& D.V. Evans in J. Fluid Mechanics in 1979, Vol. 90, pp. 337-362. We too found that two barriers could be very close and still give accurate results on a wide-spacing approximation. But for very closely-spaced barriers there is always going to be a resonance close to $\mathrm{Ka}=1$ due to the solid body-like flow of the trapped fluid and this does not occur if the bodies are in contact.

So whereas over most of the frequency spectrum, the method might work, there will always be problem at some frequencies.

\section{Author's reply:}

Thank you for the comment. I will look into how the resonance you indicated may affect the conclusions I presented in the Workshop.

Discusser: J.N. Newman

Do you have any ideas why the errors are relatively large for heave and small for surge?

\section{Author's reply:}

Suppose that we calculate the hydrodynamic forces acting on a box-type floating structure by dividing the structure into two substructures and applying the hydrodynamic interaction theory of Kagemoto \& Yue, then there exists some part of the substructures where the effect of the waves coming from the other substructure is not properly accounted for because the precondition of the Bessel functions' addition theorem is not satisfied. As I presented in the Workshop, in the case of two very closely located vertical plates of zero thickness, the part where the precondition of the Bessel functions' addition theorem is violated is the two vertical surfaces of the plates facing each other. Then, even if the effect of the waves coming from the other plate is not properly accounted for on the two vertical surfaces, the errors for the surge force due to this fact should be very small (or even zero if the distance between the two plates is zero), because the errors caused by the wrong estimation of the wave effects on the two vertical surfaces cancel each other. On the other hand, however, if we consider two closely located thick plates (box-type substructures), then the part where the precondition of the Bessel functions' addition theorem is violated also includes some part of the bottom surfaces, which in turn implies the pressures acting on the corresponding bottom surface are wrongly estimated and therefore the errors for the resultant heave forces could be significant.

\section{Discusser: J.N. Newman}

The "pumping" resonance in the gap will occur at $K^{6}$ times draft $=1$. Is that in the range of your tests or outside that range? 


\section{Author's reply:}

It is not in the range of our tests. However, I recently carried out experiments using two vertical plates in which I saw what happened if the distance of the two plates was very small, and thus $\mathrm{K} *$ draft $=1$. So far I have not observed strong resonant motions of the water column in the gap nor a complete reflection. 